\title{
THE VOYAGER ENCOUNTER WITH URANUS
}

E. C. Stone*

California Institute of Technology

Pasadena, California 91125

The Voyager 2 spacecraft is targeted for an encounter with Uranus in January, 1986. In addition to a brief description of the 11 scientific investigations and the Uranian encounter geometry, the scientific capabilities of Voyager 2 are discussed for the general areas of the atmosphere, the rings, the satellites, and the magnetosphere.

Introduction

In 1977, NASA launched two Voyager spacecraft designed for the study of Jupiter and Saturn and the interplanetary medium at increasing distances from the sun. The trajectory of Voyager 2 was chosen to include the option for continuing on to Uranus and possibly Neptune. The Voyager 2 closest approach to Saturn occurs on August 26, 1981, followed by a closest approach to Uranus on January 24, 1986. The flyby of Uranus has been designed so that the Voyager 2 spacecraft can continue on to an encounter with Neptune on August 24, 1989. Detailed information on the primary mission, on the spacecraft, and on each of the 11 investigations is available in two special issues of Space Science Reviews (21, 75$376,1977)$. The general capabilities of the Voyager investigations at Uranus will be similar to those at Jupiter and Saturn which are reported in detail in Science (204, 945-1007, 1979; 206, 925-995, 1979; and 212, 159-243, 1981).

There are 11 scientific investigations on Voyager 2 (see Table 1) involving about 120 scientific investigators. The nominal characteristics of the instruments are contained in Table 2, while Figure 1 illustrates the location of the instruments on the Voyager spacecraft. The four boresighted, remote-sensing instruments (Imaging Science, Infrared Spectroscopy and Radiometry, Photopolarimetry, and UItraviolet Spectroscopy) share the scan platform which

* Voyager Project Scientist 
has two axes of articulation in order to provide complete angular coverage.

Due to the greater distance to Uranus, the spacecraft data rates will necessarily be lower than at Saturn. It is currently planned, however, to reprogram the spacecraft to perform onboard image compression and to use an efficient hardware encoder in order to return $\sim 440$ images/day, comparable to that achieved at Saturn. Mission Characteristics

Figure 2 provides an ecliptic plane projection of the trajectories of Voyager 1 and Voyager 2. At the time of the Voyager 2 encounter, Uranus' spin axis will be directed essentially toward the sun, resulting in the appearance of the Uranian satellite and ring system as a bull's eye to the approaching spacecraft. The view normal to the trajectory plane (Figure 3 ) shows this unique encounter geometry. Figure 3 also indicates that the spacecraft disappears behind Uranus as viewed both from the sun and from the Earth, so that both a solar and an Earth occultation experiment can be performed on the rings and on the planet. The geometry of the radio occultation experiments on the rings and on the planet as viewed from Earth is shown in Figure 4. The approach distances for the spacecraft during these events are indicated in Table 3.

Table 1. Voyager Science Investigations

\begin{tabular}{ll}
\hline Investigation Area & Principal Investigator/Institution \\
\hline Imaging Science (ISS) & Smith/Univ. Arizona (Team Leader) \\
Infrared Spectroscopy and Radiometry (IRIS) & Hanel/GSFC \\
Photopolarimetry (PPS) & Lane/JPL \\
Ultraviolet Spectroscopy (UVS) & Broadfoot/Univ. So. California \\
Radio Science (RSS) & Tyler/Stanford Univ. (Team Leader) \\
Magnetic Fields (MAG) & Ness/GSFC \\
Plasma (PLS) & Bridge/MIT \\
Plasma Wave (PIS) & Scarf/TRW \\
Planetary Radio Astronomy (PRA) & Warwick/Radiophysics, Inc. \\
Low Energy Charged Particles (LECP) & Krimigis/JHU/APL \\
Cosmic Rays (CRS) & Vogt/Caltech
\end{tabular}


Table 2. Instrument Characteristics

Investigation Nominal Characteristics

ISS

Two Se-S vidicon cameras ( $f=1500 \mathrm{~mm}$ and $f=200 \mathrm{~mm})$; Narrow-angle camera: $19 \mu \mathrm{rad} / 7$ ine pair, 2900 $6400 \AA$

IRIS Michelson interferometer $(3.3-50 \mu \mathrm{m})$ and radiometer $(0.33-2 \mu \mathrm{m}) ; 51 \mathrm{~cm}$ telescope; $0.25^{\circ}$ FOV

PPS Photomultiplier with $15 \mathrm{~cm}$ telescope; 2630 - 7500 $\AA$; $3.5^{\circ}, 1^{\circ}, 1 / 4^{\circ}, 1 / 10^{\circ}$ FOV; 2 linear polarizers

UVS

Grating spectrometer; $500-1700 \AA$ with $10 \AA$ resolution; airglow $\left(1^{\circ} \times 0.1^{\circ} \mathrm{FOV}\right)$ and occultation $\left(1^{\circ} \times 0.3^{\circ} \mathrm{FOV}\right)$

RSS

S-Band (2.3 GHz) and X-band (8.4 GHz); U1tra

Stable Oscillator $\left(<4 \times 10^{-12}\right.$ short-term drift)

MAG Two low-field $\left(<10^{-6}-0.5 \mathrm{G}\right)$ and two high-field (5x10-4 - 20 G) magnetometers; $13 \mathrm{~m}$ boom;

$0-16.7 \mathrm{~Hz}$

PLS Earth-pointing sensor (10 eV - $6 \mathrm{keV}$ ions) and lateral sensor (10 eV - $6 \mathrm{keV}$ ions, $4 \mathrm{eV}-6 \mathrm{keV}$ electrons)

PWS

Sixteen channels $(10 \mathrm{~Hz}-56.2 \mathrm{kHz})$; waveform analyzer $(150 \mathrm{~Hz}-10 \mathrm{kHz})$; share PRA antennas

PRA

Stepping receiver $(1.2 \mathrm{kHz}$ and $20.4 \mathrm{kHz}-40.5$

$\mathrm{MHz}$; right and left circular polarization; orthogonal $10 \mathrm{~m}$ monopole antennas

LECP

Two solid-state detector systems on rotating platform; $10 \mathrm{keV}$ - $10 \mathrm{MeV}$ electrons; $10 \mathrm{keV} /$ nuc - $150 \mathrm{MeV} /$ nuc ions

CRS

Multiple solid-state detector telescopes; 3 - 110 MeV electrons; $\sim 1$ - $500 \mathrm{MeV} /$ nuc nuclei; 3-dimensional anisotropies

Atmospheric Studies

The dynamics, structure, and composition of the Uranian atmosphere will be studied by Voyager 2. One of the important factors for dynamics studies is the expected resolution of the imaging system as a function of time. Figure 5 shows an estimate of the time prior to closest approach during which the planet can be imaged with a given resolution. Also shown is the size of the infrared and photopolarimeter fields of view. About 700 hours before encounter, the resolution of the narrow-angle camera will exceed $700 \mathrm{~km} / \mathrm{l}$ ine pair. Since this is the expected resolution 


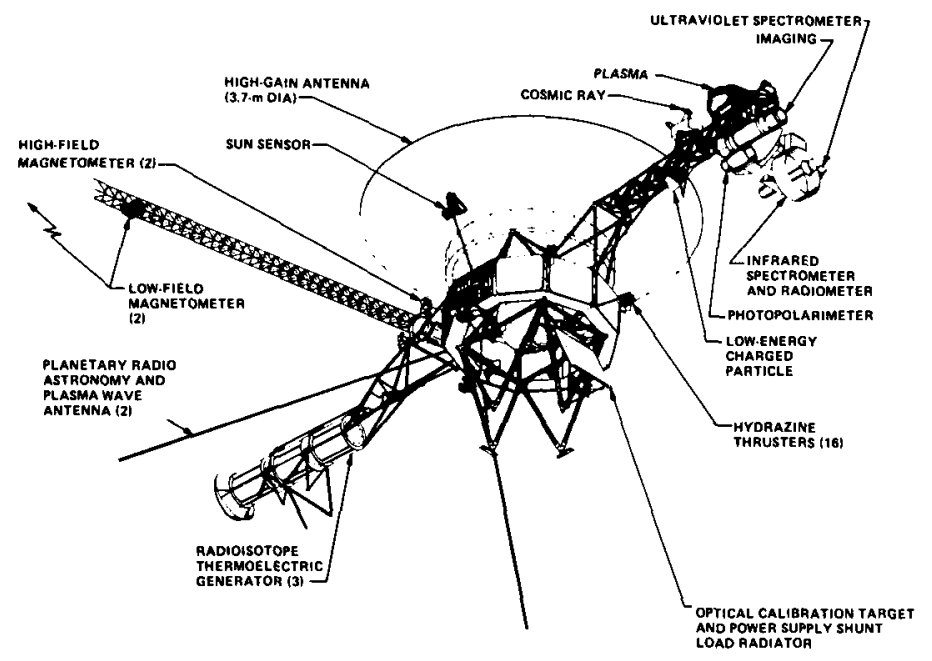

Figure 1. A drawing of the Voyager spacecraft showing the location of the science instruments. The Radio Science Investigation uses the dual-frequency spacecraft transmitters, an U1tra Stable Oscillator, and the 3.66-m parabolic antenna.

from the Space Telescope in orbit about Earth, Voyager 2 wi11 exceed the resolution capability of Space Telescope for a period of about 700 hours during both pre- and post-encounter. The wideangle camera will exceed the $700 \mathrm{~km} / \mathrm{line}$ pair resolution during a period \pm 4 days around closest approach.

Examples of Saturn images with a resolution of $\sim 700 \mathrm{~km} / 1$ ine pair are shown in Figures $6 a$ and $6 b$, one taken through a violet filter and the other through a green filter. These images demonstrate the scale of cloud structure that may be visible at this resolution, provided of course that the clouds in the Uranian atmosphere have any visible structure.

Near closest approach, the wide-angle camera can be used to advantage as illustrated in Figure 7 . The squares overlayed on the views of Uranus indicate the size of the wide-angle field of view which is 7.5 times larger than that of the narrow-angle camera.

An image typical of the wide-angle images to be taken near closest approach is shown in Figure 8 . This Voyager image of 


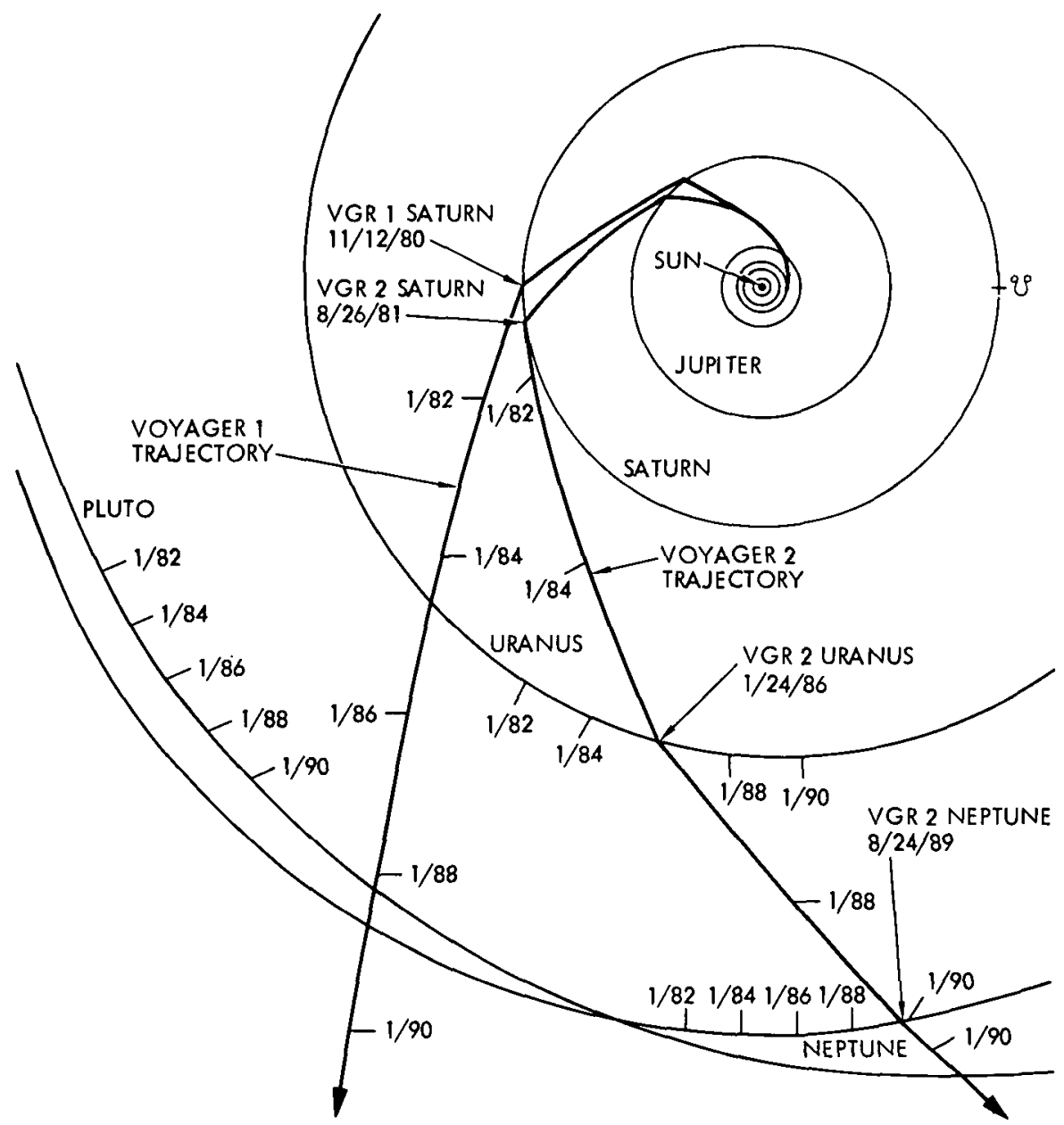

Figure 2. View from normal to the ecliptic plane of the Voyager 1 and 2 trajectories.

Saturn's south polar region was taken from a distance of about $440,000 \mathrm{~km}$ when the resolution was about $66 \mathrm{~km} / \mathrm{l}$ ine pair. A great deal of structure in the clouds can be resolved from this distance with the wide-angle camera. Even though the lighting level at Uranus is lower than at Saturn, the wide-angle camera will not be smear-limited since less than a two-second exposure with the clear filter will be required. In addition, the wide-angle camera is equipped with a narrow-band filter centered on the $541 \mathrm{~nm}$ methane 


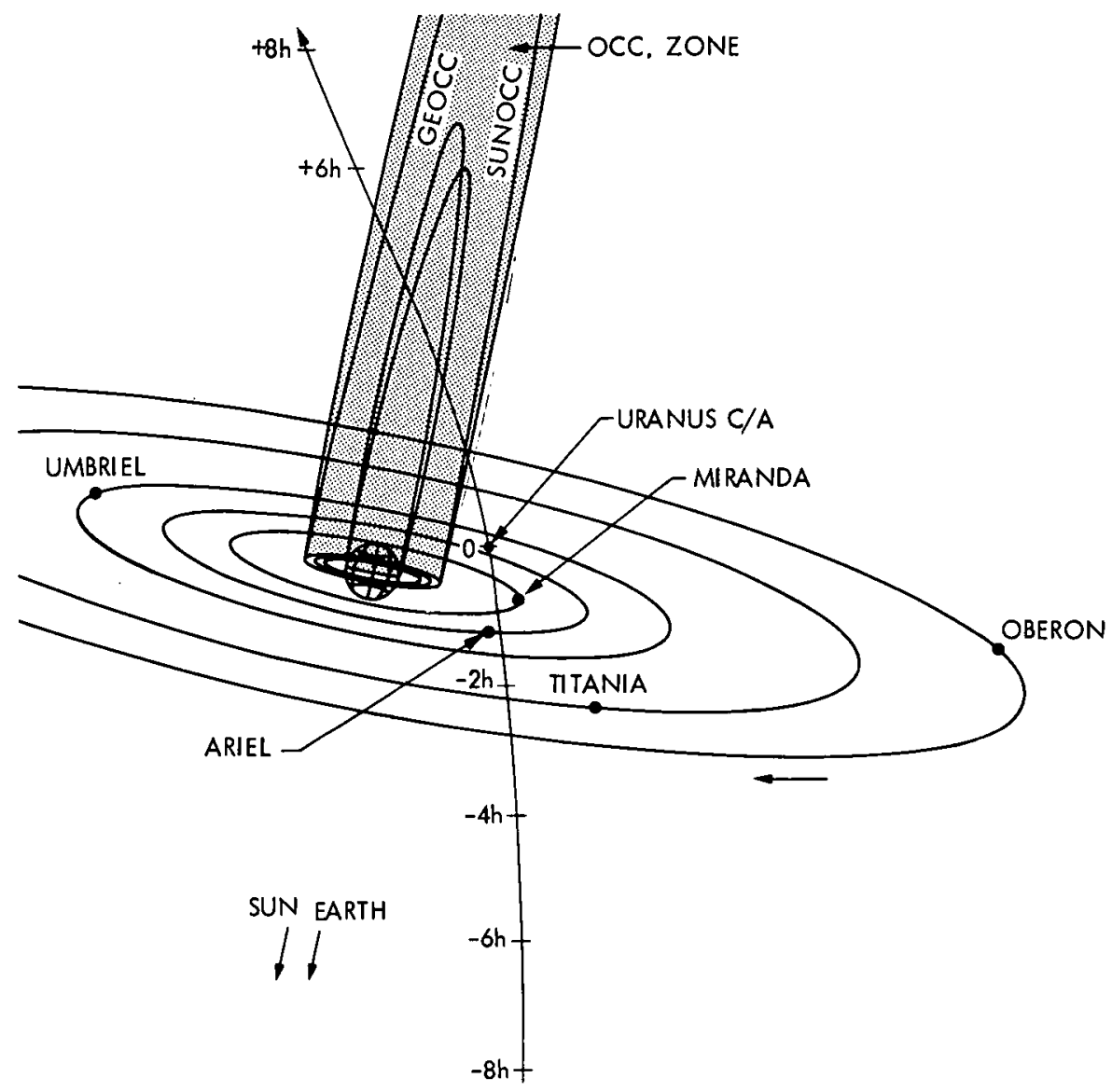

Figure 3. View from normal to the plane of the trajectory for the Voyager 2 Uranus encounter. The regions in which Earth (GEOCC) and sun (SUNOCC) occultations occur are shown.

absorption line where some contrast may be expected.

Atmospheric winds can also be derived from a latitude temperature profile of the atmosphere as has been done' at Jupiter and Saturn. The size of the infrared field of view is indicated in Figure 5 .

The study of atmospheric structure and composition will be addressed by the infrared, radio science, and ultraviolet investigations. The atmospheric temperature/pressure profile will be derived from the radio science occultation experiment and by 


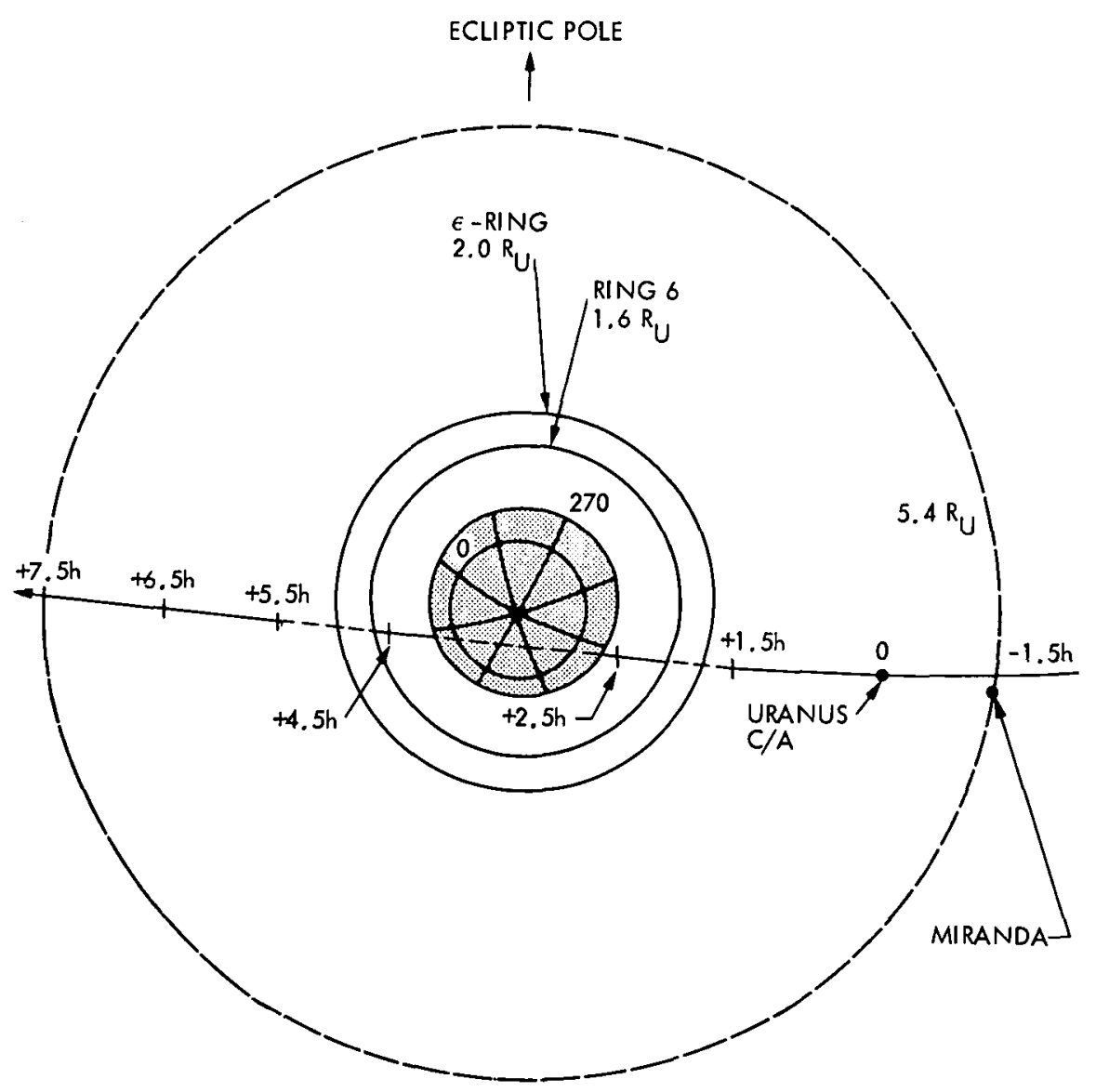

Figure 4. View from Earth of the Voyager 2 Uranus encounter. Only the inner and outermost known rings are indicated. Radio occultation studies will occur as the spacecraft passes behind the rings and the planet.

infrared temperature sounding measurements. The scale heights of $\mathrm{H}_{2}$ and $\mathrm{CH}_{4}$ in the upper atmosphere will be determined by the ultraviolet solar occultation measurement, while the same instrument will derive the relative abundances of $\mathrm{H}$ and $\mathrm{He}$ through airglow measurements.

The infrared measurements are particularly difficult because Uranus is so much colder than Jupiter and Saturn. The IRIS instrument does, however, have adequate sensitivity in the critical 
Table 3. Voyager 2 Uranus Encounter

Time, Closest Approach

(Spacecraft Event Time, GMT)

Radius, Closest Approach

Radius, Ring Plane Crossing

Distance $\left(10^{3} \mathrm{~km}\right)$ Earth Occultation
January 24, 1986 19:00:00.0

$107,080 \mathrm{~km}$

$115,200 \mathrm{~km}$

137

155

186

255

280

298

$\begin{array}{cl}\text { Distance }\left(10^{3} \mathrm{~km}\right) & \text { Uranus entrance } \\ \text { Sun Occultation } & 177 \\ \text { Uranus exit } & 240\end{array}$

wavenumber range between 200 and 500 wavenumbers (20 to 50 microns). In this wavelength interval, the pressure-induced $S(0)$ line of $\mathrm{H}_{2}$ can be used for the temperature/pressure profile analysis and is also the region in which He abundance can be inferred from the details of the $\mathrm{H}_{2}$ absorption line.

The sensitivity of the infrared instrument is indicated in Figure 9. A single interferogram is accumulated over a 48-second interval and, as shown in Figure 9, will have a 10:1 signal-tonoise at $200 \mathrm{~cm}^{-1}$ and will have about $1: 1$ signal-to-noise at $\sim 350$ $\mathrm{cm}^{-1}$. Also indicated are the increased signal-to-noise ratios which are possible with one-hour averaging and with one-day averaging during the encounter phase. With the 24-hour average spectrum, the range should extend to $\sim 450 \mathrm{~cm}^{-1}$, with a signal-to-noise of better than 100 at $200 \mathrm{~cm}^{-1}$. With this sensitivity, it should be possible to derive a temperature/pressure profile between approximately 80 and 1000 millibars (Hanel, private communication).

Although most of the thermal emission from Uranus lies below the $500 \mathrm{~cm}^{-1}$ limit of the infrared instrument, a model-dependent extrapolation can be used to estimate the thermal flux at lower wavenumbers. The radiometer portion of the instrument will provide 


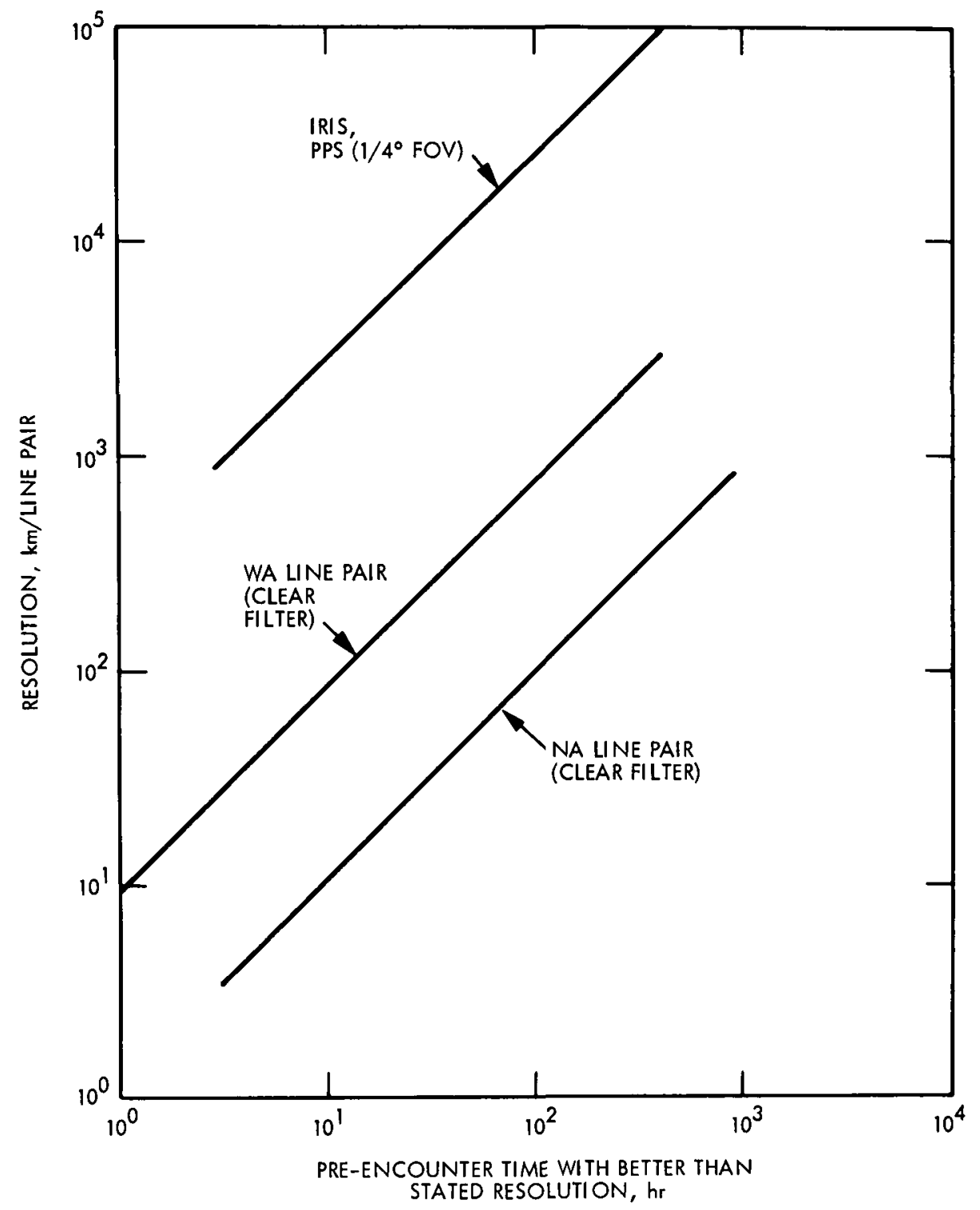

Figure 5. Resolution of the narrow-angle camera (NA), the wideangle camera (WA), and the size of the IRIS and PPS fields of view as a function of time before encounter.

the phase function of the reflected light, as well as the geometrical and Bond albedo. The ultimate accuracy to which the energy 
(a)

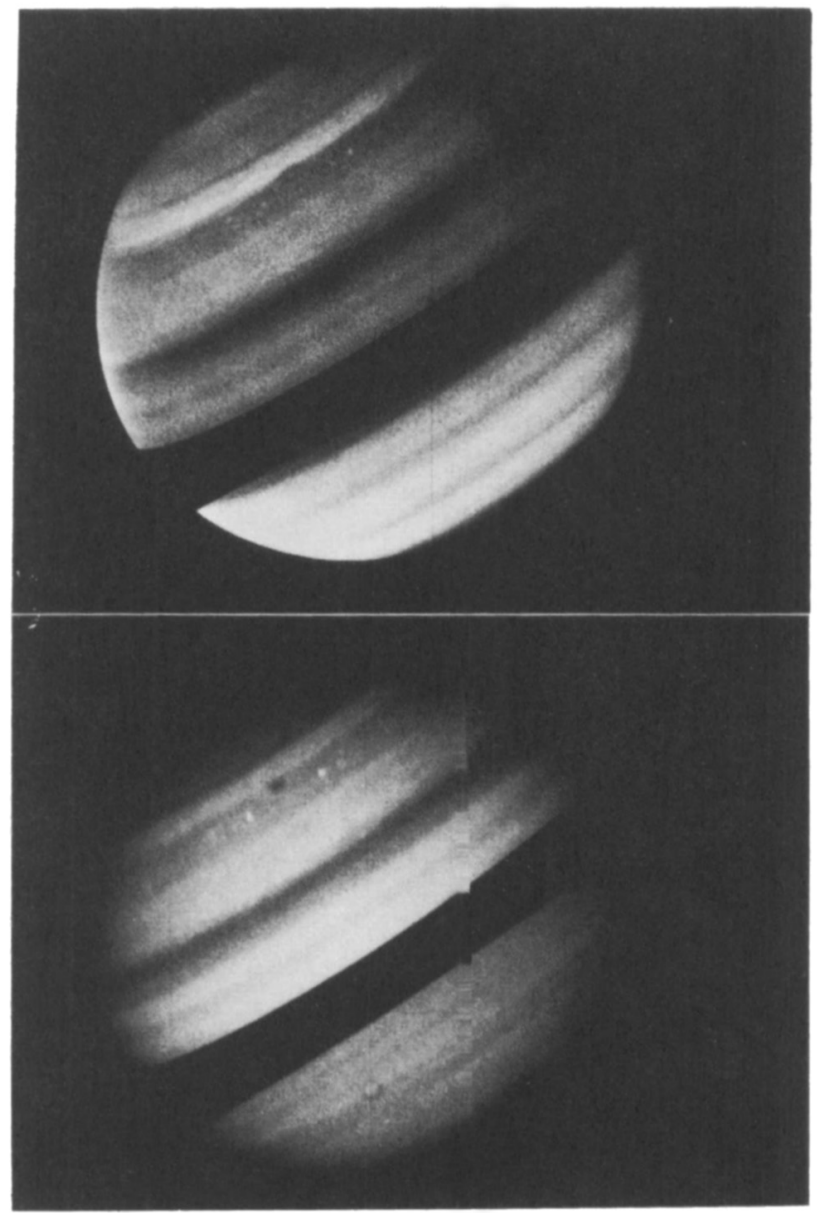

Figures $6 \mathrm{a}$ and $6 \mathrm{~b}$. Two images of Saturn taken with the narrow-angle camera 25 days before closest approach. The resolution is $\sim 700 \mathrm{~km} /$ line pair. Figure a was taken through a violet filter and Figure $b$ through a green filter. Discrete cloud structures are visible at $\sim 40^{\circ} \mathrm{N}$. The large dark spot at $\sim 45^{\circ} \mathrm{N}$ in the green image is elongated in the violet.

balance of Uranus can be determined has not been assessed. Internal Structure

As we have heard at this conference, models of the internal structure of Uranus depend upon accurate values for a number of observable parameters, such as the oblateness of the planet, the 

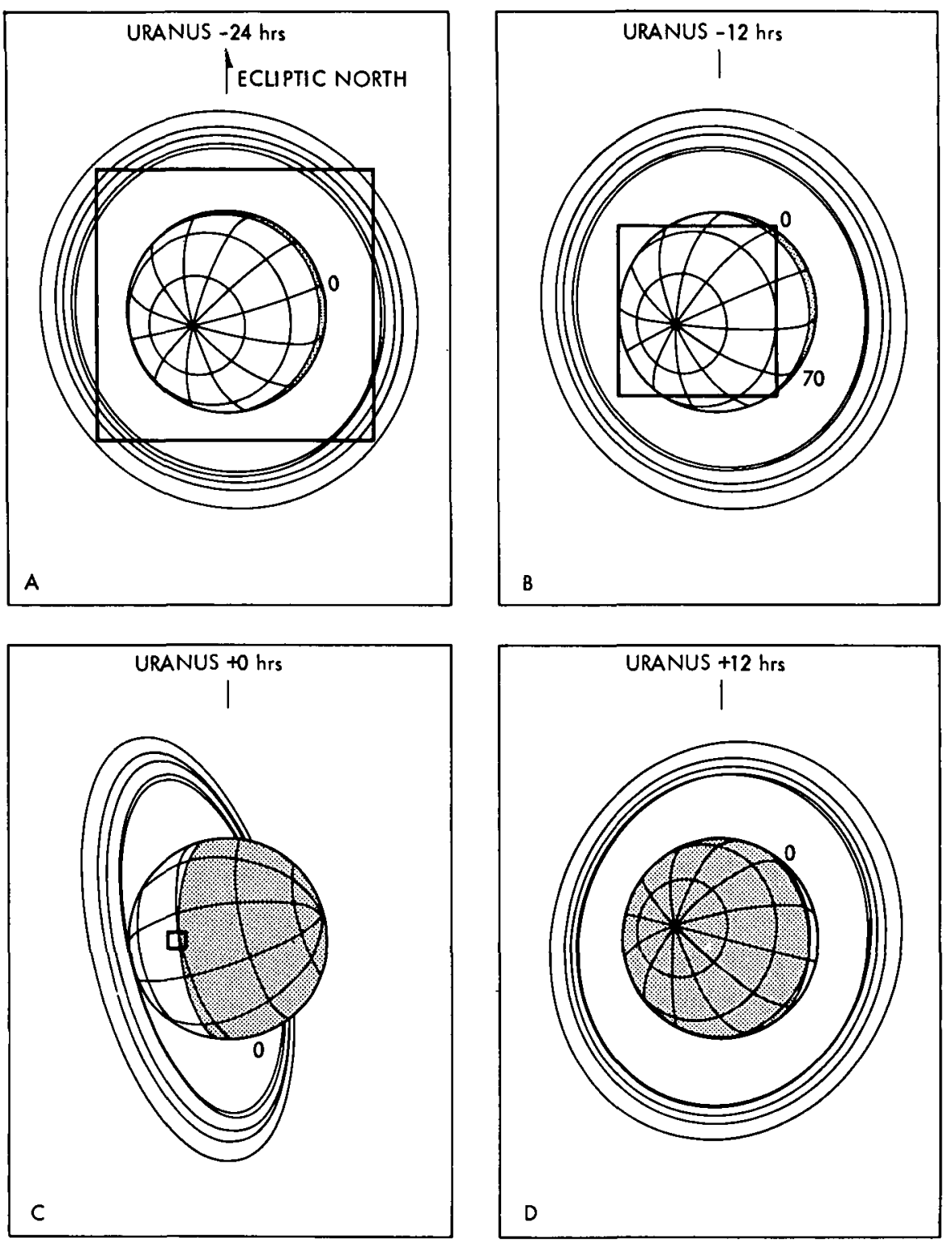

Figure 7. Views of Uranus from the spacecraft at 12-hour intervals. The square overlay indicates the wide-angle field of view which is 800 picture elements on a side. 


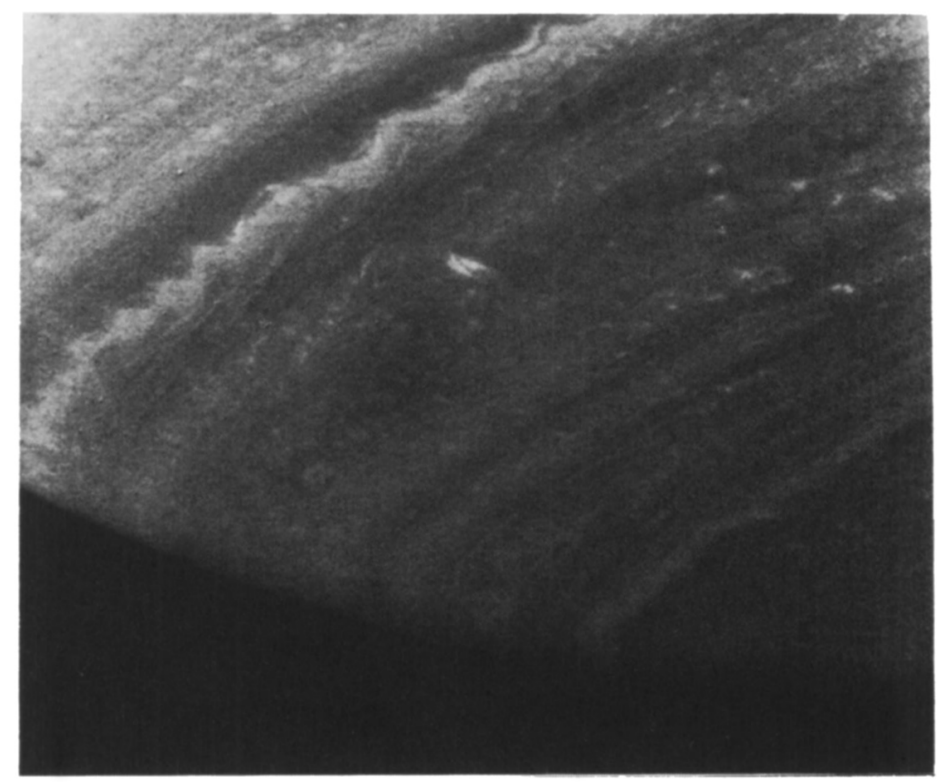

Figure 8. This image of Saturn's south polar region was taken with the wide-angle camera from a distance of $442,000 \mathrm{~km}$. The resolution is $\sim 66 \mathrm{~km} / \mathrm{line}$ pair. Waves and eddies are evident in the large-scale light and dark bands.

zonal harmonics of the Uranian gravity potential, and the period of rotation. Currently, the best estimate of the $\mathrm{J} 2$ harmonic of the gravitational potential is $3352 \pm 5 \times 10^{-6}$, which has been derived by Elliot, et al. (Astrophys. J., 86, 444-455, 1981) from the rate of precession of the eccentric rings around Uranus. Voyager 2 can provide an independent measure of 32 by an accurate determination of the spacecraft trajectory during encounter. It is estimated that the uncertainty of the Voyager 2 determination of 32 will be approximately $\pm 30 \times 10^{-6}$ (J. D. Anderson, private communication). Elliot, et al. have also determined a value of $-29 \pm 13 \times 10^{-6}$ for the 34 gravitational harmonic. Voyager 2 will be able to contribute to the determination of this value through studies of the eccentric rings. However, the uncertainty of the value of $\mathrm{J4}$ derived from the trajectory information will be $\pm 500 \times 10^{-6}$, too 
uncertain to significantly contribute to a more accurate determination of 34 .

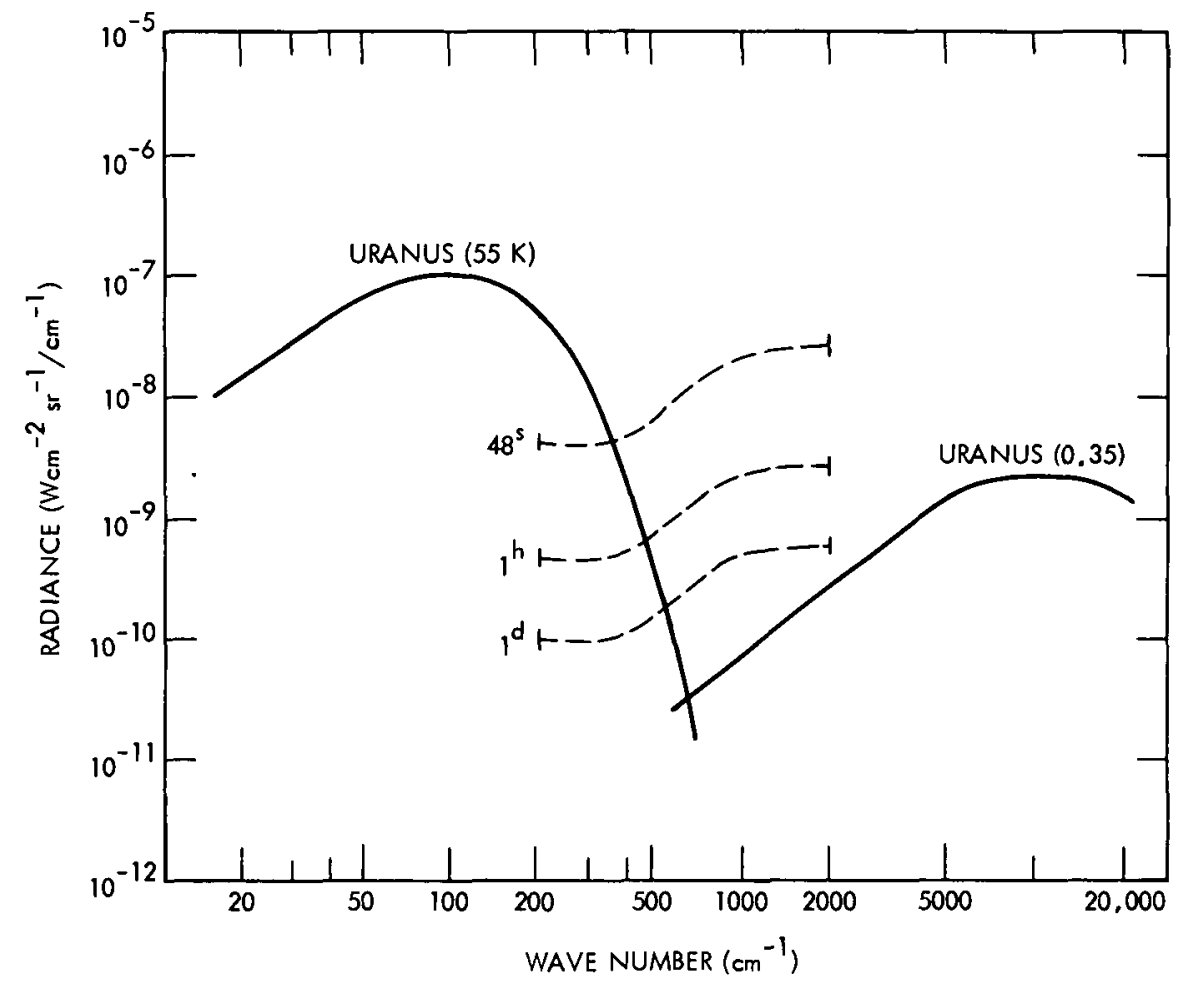

Figure 9. The emitted and reflected radiance levels for Uranus are indicated schematically. The noise-equivalent-radiance for the infrared interferometer is indicated for a single interferogram (48 seconds) and for one-hour and one-day averages.

The radio occultation experiment should provide an accurate measurement of the equatorial radius of the planet and, therefore, provide additional information on that critical parameter.

There are several possibilities for measuring the rotation period for the planet for which current values range from 16 to 24 hours. If Uranus has a magnetic field, it will presumably rotate with the interior of Uranus and it is likely that there will be a corresponding periodicity in the radio emission from the planet 
which will be recorded by the Planetary Radio Astronomy receiver. Time-lapse imaging of the rotational pole of the atmosphere may also yield estimates of the period of rotation, provided there are discrete azimuthal asymmetries or longitudinal structures in the clouds. of course, the period of the clouds need not be identical to the internal period of the planet.

The Rings

Several investigations will contribute to studies of the rings. The photopolarimeter will undertake stellar occultation studies, the radio experiment will perform radio occultation studies, and the ultraviolet spectrometer will undertake solar occultation studies of the rings, while the imaging system will directly image the rings.

Although there has been no search for the stellar occultations which will occur during the Uranus encounter, it seems reasonable that there will be a number of opportunities similar to those at Saturn, where the photopolarimeter on Voyager 2 will observe stellar occultations by the rings of $\delta$ Scorpii and $B$ Tauri. The $\delta$ Scorpi $i$ occultation should provide $\sim 300$ meter resolution of the Saturnian rings with 10:1 signal-to-noise, and the $B$ Tauri occultation should be about five times better. It is interesting to note that the width of the Fresnel Zone for the Voyager 2 observations will be about 15 meters, so that the observations will be primarily limited by the $10 \mathrm{millisecond} \mathrm{sampling} \mathrm{time} \mathrm{of} \mathrm{the} \mathrm{instrument.}$

The radio occultation studies of the rings will be performed both at $X$-band and $S$-band, that is, at wavelengths of about 3.6 and about $13 \mathrm{~cm}$. The Fresnel Zones for these two wavelengths will be 3 to $5 \mathrm{~km}$, while the footprint of the $X$-band antenna beam on the Uranian ring plane will be approximately $200 \mathrm{~km}$.

It may also be possible to perform a solar occultation study of the rings using the ultraviolet spectrometer. With the geometry of our flyby trajectory, the size of the sun corresponds to a distance of about $60 \mathrm{~km}$ in the ring plane.

The imaging studies of the rings will occur during both approach and post encounter. During the approach phase, the resolution may be smear-limited to $\sim 30 \mathrm{~km}$, although a detailed analysis 
of the optimum observing strategy has not been undertaken. If the Jovian ring and the F-ring at Saturn are any indication, the narrow rings around Uranus may well have a substantial component of very fine material and may be much brighter in forward-scattered light than in back-scattered. In that case, the best images of the narrow rings may occur after closest approach when shorter exposures can be taken, thereby reducing the smear.

In addition to studying the rings themselves, there will be a systematic search for shepherding satellites which may be responsible for the stability of the narrow rings at Uranus, much as two shepherding satellites are responsible for the stability of the Fring at Saturn. It is also possible that there will be additional material around Uranus which can be observed from Voyager 2 but has not yet been detected from Earth.

The Satellites

Studies of the five known satellites of Uranus will also be a principal objective of the Voyager encounter. The flyby distances for each of the satellites are indicated in Table 4. Also indicated is the range of expected imaging resolution on each of the satellites. The actual resolution will be determined by the extent to which spacecraft maneuvers can compensate for the smearing effects due to the velocity of the spacecraft. Since the detailed encounter sequence design has not been done, it is unknown how well such maneuvers can be implemented. However, the technique of image motion compensation was successfully demonstrated during the Voyager 1 encounter with Rhea. With image motion compensation the resolution on Miranda could be better than $1 \mathrm{~km} / \mathrm{l}$ ine pair, comparable to the best resolution of the Galilean satellites and the intermediatesized icy satellites at Saturn.

Other investigations will also contribute to the satellite studies. The flyby distances are close enough for Miranda and Ariel to permit infrared measurements of their thermal characteristics. The photopolarimeter will determine the scattering characteristics of the icy surfaces as a function of phase angle for both ultraviolet and the far red end of the spectrum. In addition, it may be possible to search for an atmosphere of one of the satellites if 
there is an appropriate stellar occultation.

Table 4. Satellite Encounters

\begin{tabular}{lccc}
\hline & $\begin{array}{c}\text { Closest Approach } \\
\text { Satellite }\end{array}$ & $\left(10^{3} \mathrm{~km}\right)$ & \multicolumn{2}{c}{ Resolution* } & Gm/line pair) \\
\hline U1 Miranda & 16 & $<1$ & $\sim 15$ \\
U2 Ariel & 140 & 3 & $\sim 30$ \\
U3 Umbriel & 326 & 7 & $\sim 50$ \\
U4 Titania & 372 & 7 & $\sim 50$ \\
U5 Oberon & 471 & 9 & $\sim 50$ \\
\hline
\end{tabular}

* Actual resolution will depend upon the extent to which a manoeuver can compensate for the velocity of the spacecraft relative to the satellite.

\section{The Magnetosphere}

As discussed by Axford at this conference, it is unknown whether or not Uranus has a magnetic field. If Uranus does have a magnetic field giving rise to non-thermal radio emissions, the Planetary Radio Astronomy experiment may provide the first answer to this basic question. In addition, any one of the fields and particles instruments can directly detect the presence of a magnetic field once the spacecraft enters the Uranian magnetosphere. It should be possible to determine not only whether or not there is a magnetic field, but also the orientation of the dipole axis with respect to the spin axis, the strength of the magnetic field, and the period of rotation.

The Voyager 2 instruments should also provide broad coverage of the plasma, energetic particle, and wave properties of a Uranian magnetosphere. Studies of this potentially unique magnetosphere may be of special significance because it is possible that the solar wind will be impinging on one of the magnetic polar regions.

Although there have been no detailed calculations of what such an interaction might be like, it is certainly plausible that the solar wind plasma could penetrate deeply into the polar region of the 
Uranian magnetosphere.

As Axford has pointed out, the Uranian magnetosphere may also be a quiet magnetosphere because of the smaller energy input from the solar wind, in which case the absorption lanes in the trapped radiation environment resulting from the interaction of the satellites and the rings could be quite pronounced. Assuming that the dipole axis is colinear with the spin axis of the planet, the Voyager 2 spacecraft will penetrate into an L-shell of about 4.5 Uranian radii, crossing the magnetic shells associated with the five known Uranian satellites and permitting studies of the satellitemagnetosphere interactions.

Conclusion

This is necessarily a rather preliminary overview of the Voyager 2 capabilities at Uranus. Detailed planning of the sequence of observations will begin in 1984, at which time it will be possible to assess more precisely the potential scientific return from the Voyager 2 encounter with Uranus. It is also likely that new information from continued Earth-based observations of Uranus and possibly from Space Telescope will influence the design of the Voyager 2 encounter sequence. I think it is quite appropriate, however, that in this 200th anniversary of the discovery of Uranus we should be considering the possibility of the first visit to that planet by a spacecraft from Earth.

\section{Acknowledgements}

The Voyager Project is being carried out for the National Aeronautics and Space Administration by the Jet Propulsion Laboratory of the California Institute of Technology under Contract NAS7-100. 